\author{
Anna Ignaczak ${ }^{1,2}$, Elektra Szymańska-Garbacz ${ }^{3,4}$, Ewa Kwiecińska5 ${ }^{5}$ Leszek Czupryniak $^{6}$ \\ ${ }^{1}$ Department of Nursing with Nursing Practice Laboratories, Chair of Nursing Education, Medical University of Lodz \\ ${ }^{2}$ Department of General and Oncological Gastroenterology, Barlicki University Hospital No. 1, Medical University of Lodz \\ ${ }^{3}$ Department of Infectious and Liver Diseases, Medical University of Lodz \\ ${ }^{4}$ Department of Internal Medicine and Nephrodiabetology, Medical University of Lodz \\ ${ }^{5} 2^{\text {nd }}$ Department of Internal Medicine, District Hospital, Konin \\ ${ }^{6}$ Department of Diabetology and Internal Diseases, Medical University of Warsaw
}

\title{
Risk factors for hypoglycaemia in in-patients with diabetes treated with continuous insulin intravenous infusion
}

\section{ABSTRACT}

Introduction. Hypoglycaemia is the most frequent complication of diabetes therapy. It leads to unpleasant symptoms and, if severe may, result in coma and even death. Hospitalized patients treated with intravenous insulin therapy are at particularly high risk of hypoglycaemia. Nursing staff play crucial role in preventing, early detecting and treatment of hypoglycaemia caused by insulin given intravenously.

Material and methods. This observational, prospective and non-interventional study aimed at assessing prevalence and risk factor of hypoglycaemia during continuous intravenous insulin infusion (CIVII) in a hospital setting. Two hundred consecutive patients (48 with type 1 diabetes and 152 with type 2 diabetes) were enrolled into the study. Mean age of type 1 diabetes patients was $38 \pm 14$ years, and those with type 2 diabetes $61 \pm 12$ years $(p<0.0001)$, and their $\mathrm{HbA}_{1 \mathrm{c}}$ was $10.1 \pm 2.9$ and $10.1 \pm 2.3 \%$, respectively. Continuous intravenous insulin infusion was given for $2.5 \pm 1.1$ days (basal infusion and three 90-min prandial boluses) according to standard protocol.

Results. Hypoglycaemia was noted in $48 \%$ of patients with type 1 diabetes and in $20 \%$ of those with type 2 diabetes ( $p<0.001$ ), most often in the second day

Address for correspondence:

prof. dr hab. n. med. Leszek Czupryniak

Klinika Diabetologii i Chorób Wewnętrznych WUM

ul. Banacha 1a, 02-097 Warszawa

Phone: +48225992583

e-mail: leszek.czupryniak@wum.edu.pl

Translation: lek. Małgorzata Kamińska

Clinical Diabetology 2017, 6, 2, 41-47

DOI: $10.5603 /$ DK.2017.0008

Received: 14.05.2017

Accepted: 01.06.2017 of CIVII. In type 1 diabetes, the main risk factor for hypoglycaemia while on CIVII was diabetes duration (the longer duration, the higher the risk) and in type 2 diabetes - daily insulin dose, total and per kg of body weight (the lower the dose, the higher the risk). Conclusions. Continuous intravenous insulin infusion should be used with utmost care in type 1 diabetes patients with long duration of the disease and in those type 2 diabetes patients who show signs of low insulin resistance (little overweight, low insulin requirement). (Clin Diabetol 2017; 6, 2: 41-47)

Key words: hypoglycaemia, insulin therapy, risk factors, hospitalization

\section{Introduction}

Diabetes patients usually require temporary hospital treatment in the course of the disease. The most common causes of hospitalization of patients with diabetes are: acute complications of diabetes (coma, ketoacidosis), exacerbation of chronic complications (myocardial infarction, stroke, diabetic foot syndrome), significant metabolic decompensation of diabetes requiring intravenous insulin, or other acute diseases (infections, diseases requiring surgical treatment) [1-3].

The most frequent complication of diabetes treatment is hypoglycaemia, currently defined as glucose $<70 \mathrm{mg} / \mathrm{dL}$ [4]. About $30 \%$ of young patients on insulin therapy experience diabetes coma in their lives; this complication develops in about $10 \%$ of insulin-treated patients yearly. Recurrent coma affects about $3 \%$ of patients. Hypoglycaemic coma causes about $3-4 \%$ of diabetes-related deaths [5]. 
Because of short- and long-term adverse effects of hypoglycaemia, it is now recommended that the treatment of diabetes should be associated with the lowest possible risk $[4,6]$. Continuous intravenous insulin infusion (CIVII), routinely used in hospital settings in patients with acute or chronic metabolic decompensation of diabetes, can easily lead to hypoglycaemia. During hospitalization, the intravenous administration of insulin as well as monitoring of intravenous insulin infusion and the patient condition are at the responsibility of the nursing team.

The aim of the study was to determine the incidence of hypoglycaemia and its risk factors during intravenous insulin therapy. Identifying risk factors for developing hypoglycaemia will reduce the risk of this serious complication in hospitalized patients.

\section{Patients}

The study was prospective, observational and non-interventional. An analysis was performed of intravenous insulin therapy in 200 consecutive diabetic patients hospitalized at the Barlicki Memorial Teaching Hospital No. 1 in Lodz in whom intravenous infusion of short-acting human insulin was administered during the hospital treatment, according to the protocol described below.

The inclusion criteria were as follows: age over 18 years, time from diagnosis of type 1 or type 2 diabetes of at least 6 months, poor metabolic control of diabetes, i.e. $\mathrm{HbA}_{1 \mathrm{c}}$ measured within 1 month before hospitalization of $>7.0 \%$ and insulin therapy in the form of CIVII during hospitalization. The exclusion criteria included: pregnancy; acute disease with severe general condition of the patient and/or extreme hyperglycaemia, including severe ketoacidosis, ketoacidosis coma, hyperosmolar hyperglycaemic state or lactic acidosis; newly diagnosed diabetes; diabetes of known cause not being type 1 or type 2 diabetes (e.g. diabetes associated with pancreatic disease, endocrine disease), and the need for steroid intake.

\section{Methods}

Clinical data regarding hospitalization and intravenous insulin treatment were prospectively collected, but the analysis was performed after the study group was completed. The process of analysing collected data and the results of the analysis did not affect patient management during hospitalization. The study was approved by the Bioethics Committee at the Medical University of Lodz and the Head of the Barlicki Memorial Teaching Hospital No. 1 in Lodz.

The following parameters were analysed: sex, age, body weight and height, body mass index (BMI), type of diabetes (type 1, type 2), time from diagnosis of diabetes, chronic complications of diabetes and concomitant diseases, antidiabetic treatment used before hospitalization, results of laboratory tests and data regarding the course of hospitalization.

Continuous intravenous insulin infusion is the procedure used in the Barlicki University Hospital Lodz for over 20 years. The original protocol was developed in Strasbourg (France) [7, 8]. The intravenous insulin infusion consisted of a basal infusion and three 90-minute prandial boluses. Short-acting human insulin was administered in $0.9 \% \mathrm{NaCl}$ solution (50 IU in $50 \mathrm{~mL}$, $1 \mathrm{IU} / 1 \mathrm{~mL}$ ) using automatic infusion pump. Initial insulin dose was established based on insulin doses used by the patient before the study. Initial basal rate (in IU/h) was calculated as $50 \%$ of the patient's daily dose of insulin divided by 24 . The remaining $50 \%$ of insulin dose were divided equally into three prandial boluses (breakfast, lunch and dinner).

Capillary blood glucose was measured every 90 min during the day and every 3 hours during the night (10.00 p.m.-7.00 a.m.), with the mean number of measurements per day of about 12. Basal infusion rate was modified according to study protocol:

- glucose level $<40 \mathrm{mg} / \mathrm{dL}$ - infusion rate reduction by $0.4 \mathrm{UI} / \mathrm{h}$;

- glucose level $40-60 \mathrm{mg} / \mathrm{dL}$ - infusion rate reduction by $0.2 \mathrm{UI} / \mathrm{h}$;

- glucose level $60-80 \mathrm{mg} / \mathrm{dL}$ - infusion rate reduction by $0.1 \mathrm{Ul} / \mathrm{h}$;

- glucose level 80-120 mg/dL - no changes in infusion rate;

- glucose level $120-150 \mathrm{mg} / \mathrm{dL}$ - infusion rate increase by $0.1 \mathrm{UI} / \mathrm{h}$;

- glucose level 150-200 mg/dL - infusion rate increase by $0.2 \mathrm{UI} / \mathrm{h}$;

- glucose level > $200 \mathrm{mg} / \mathrm{dL}$ - infusion rate increase by $0.3 \mathrm{UI} / \mathrm{h}$, and additionally a bolus of $60 \mathrm{UI} / \mathrm{h}$ for $4 \mathrm{~min}$.

Prandial boluses lasted $90 \mathrm{~min}$ and were initiated at the beginning of the meal at strictly defined time during the day: 7.30-9.00, 12.00-13.30, 19.00-20.30.

Each bolus was divided into three periods: during first $20 \mathrm{~min}$ the patient received $30 \%$ of the dose, during subsequent $20 \mathrm{~min}-50 \%$, and during remaining 50 $\min -20 \%$ of estimated insulin dose for one prandial bolus. Subsequent prandial bolus doses were estimated based on glucose-lowering effects of previous boluses.

The intravenous insulin infusion was continued until achieving sufficient metabolic control, but not less than $24 \mathrm{~h}$. Sufficient metabolic control was defined as fasting and pre-prandial blood glucose within the range of $70-120 \mathrm{mg} / \mathrm{dL}$ and blood glucose $2 \mathrm{~h}$ after a meal 


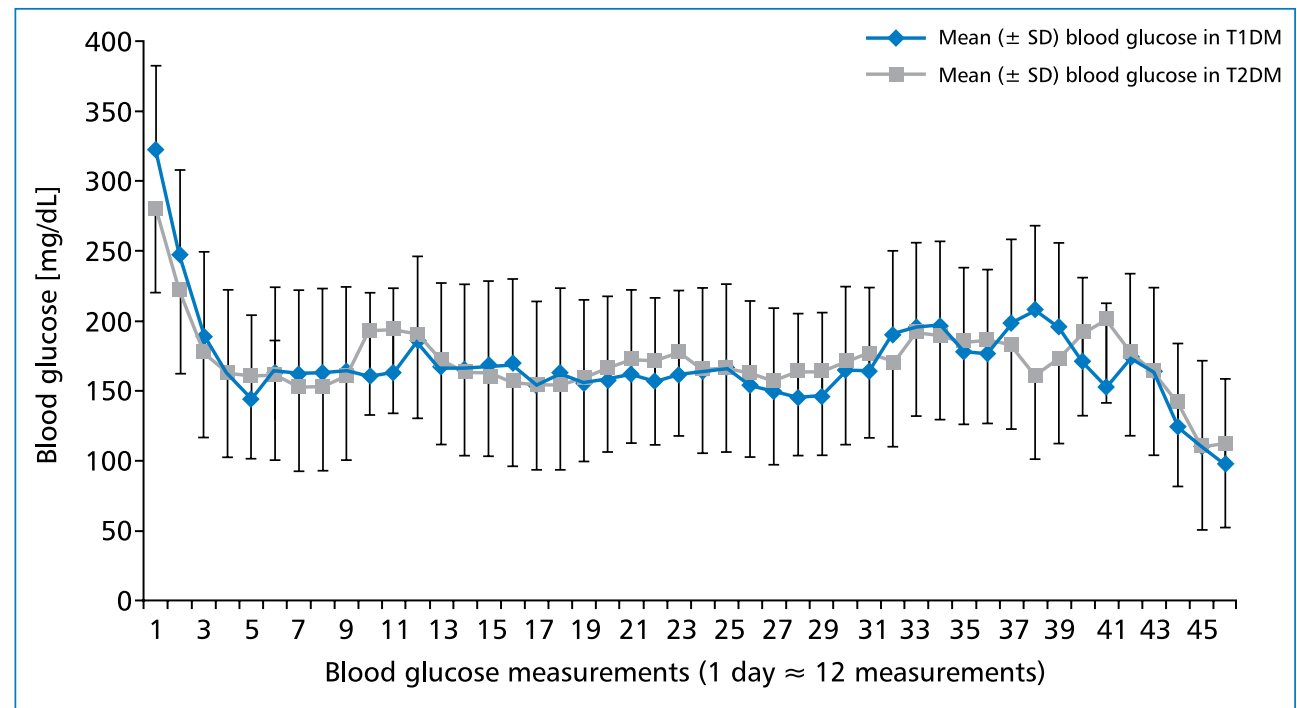

Figure 1. Mean values of blood glucose during continuous intravenous insulin infusion in patients with type 1 (T1DM) and type 2 diabetes mellitus (T2DM)

$<160 \mathrm{mg} / \mathrm{dL}$. The intravenous insulin administration regimen was the same for patients with type 1 and type 2 diabetes.

Hypoglycaemia was diagnosed in every case when measured blood glucose level was below the diagnostic threshold for hypoglycaemia of $<60 \mathrm{mg} / \mathrm{dL}$ (a value recommended in 2012, when the study was planned and its protocol developed) or when continuous infusion of insulin had to be stopped due to clinical symptoms of hypoglycaemia, regardless of the blood glucose level.

The obtained results were statistically analysed with Statistica 9.1 software. The Shapiro-Wilk test was utilized to assess the normality of data distribution. For the assessment of between-group differences in normally distributed variables, the Student's t-test for independent samples was used, and the U-Mann-Whitney test was used for non-normally distributed variables. The correlation analysis was utilized to evaluate the significance of the relationship between quantitative variables by calculating the Pearson coefficient. In order to identify risk factors for hypoglycaemia, a multiple regression model was used, defining dependent and independent variables. Statistical significance was set at $p<0.05$

\section{Results}

The study included 200 consecutively hospitalized patients who required intravenous insulin infusion: 48 patients with type 1 diabetes ( 25 women and 23 men) and 152 patients with type 2 diabetes ( 81 women and 71 men).
The mean age of the whole study group was $54 \pm$ 16 years, body weight $81.6 \pm 19.3 \mathrm{~kg}$, BMI $29.3 \pm 6.4$ $\mathrm{kg} / \mathrm{m}^{2}$, mean diabetes duration was $10.0 \pm 7.8$ years, the hospitalization time was $9 \pm 3$ days, and $\mathrm{HbA}_{1 \mathrm{c}}$ value was $10.1 \pm 2.5 \%$.

Hypoglycaemia was reported in 48 (24\%) patients. These patients had significantly longer diabetes duration (12.4 \pm 10.8 vs. $9.2 \pm 8.2$ years; $p<0.05)$ and were characterized by lower body weight (74.2 \pm 15.6 vs. $83.9 \pm 19.8 \mathrm{~kg} ; \mathrm{p}<0.01 ; \mathrm{BMI} 27.0 \pm 5.9$ vs. 30.0 $\pm 6.4 \mathrm{~kg} / \mathrm{m}^{2} ; \mathrm{p}<0.01$ ) compared with the patients in whom CIVII was not complicated by hypoglycaemia.

The mean age of the patients with type 1 diabetes was $38 \pm 14$ years and in those with type 2 diabetes it was $61 \pm 12$ years $(p<0.0001)$. Both groups of patients were not statistically significantly different in terms of time since the diagnosis of diabetes. In patients with type 1 diabetes, the duration of the disease was on average $10.4 \pm$ 8.7 years, and in the group of the patients with type 2 diabetes, the disease was diagnosed $10.2 \pm 8.4$ ago.

The patients with type 2 diabetes were significantly more likely to be obese than those with type 1 diabetes (60 vs. $15 \% ; p<0.001$ ) (Fig. 1). The BMI was $31.1 \pm$ $6.2 \mathrm{~kg} / \mathrm{m}^{2}$ in type 2 diabetic patients and $25.1 \pm 4.5$ $\mathrm{kg} / \mathrm{m}^{2}$ in type 1 diabetics $(\mathrm{p}<0.0001)$.

Table 1 presents the occurrence of diabetes complications, concomitant diseases and selected clinical parameters in patients with type 1 and type 2 diabetes.

The groups did not differ in terms of the degree of metabolic decompensation of diabetes based on the value of $\mathrm{HbA}_{1 \mathrm{c}}$ : in the patients with type 1 diabetes $\mathrm{HbA}_{1 \mathrm{c}}$ was $10.1 \pm 2.9 \%$ and in those with type 2 diabe- 
Table 1. Diabetes complications, concomitant diseases and selected clinical parameters in patients with type 1 and type 2 diabetes

\begin{tabular}{|c|c|c|c|}
\hline & $\begin{array}{c}\text { Type } 1 \text { diabetes }(n=48) \\
\text { n (\%) }\end{array}$ & $\begin{array}{c}\text { Type } 2 \text { diabetes }(n=152) \\
n(\%)\end{array}$ & p-value \\
\hline Retinopathy & $16(33 \%)$ & $63(41 \%)$ & $<0.05$ \\
\hline Nephropathy & $17(35 \%)$ & $72(47 \%)$ & $<0.05$ \\
\hline Neuropathy & $13(27 \%)$ & $40(26 \%)$ & NS \\
\hline Hypertension & $13(27 \%)$ & $120(79 \%)$ & $<0.001$ \\
\hline Previous myocardial infarction & $2(4 \%)$ & $20(13 \%)$ & $<0.01$ \\
\hline Previous stroke & $0(0 \%)$ & $14(9 \%)$ & $<0.01$ \\
\hline Liver disease & $7(15 \%)$ & $43(28 \%)$ & $<0.05$ \\
\hline Gastro-intestinal disease & $10(21 \%)$ & $31(20 \%)$ & NS \\
\hline Anaemia & $6(13 \%)$ & $13(9 \%)$ & NS \\
\hline Status post cholecystectomy & $3(6 \%)$ & $26(17 \%)$ & $<0.05$ \\
\hline Status post appendectomy & $2(4 \%)$ & $11(7 \%)$ & $<0.05$ \\
\hline \multicolumn{4}{|l|}{ Insulin therapy } \\
\hline (prior to hospitalization) & $48(100 \%)$ & $92(61 \%)$ & $<0.01$ \\
\hline \multicolumn{4}{|l|}{ Oral antidiabetic drugs } \\
\hline (prior to hospitalization) & $0(0 \%)$ & $108(71 \%)$ & $<0.001$ \\
\hline Beta-blocker therapy & $9(19 \%)$ & $60(39 \%)$ & $<0.01$ \\
\hline
\end{tabular}

NS - non-significant

tes $-10.1 \pm 2.3 \%$. Hospitalization time was similar in both groups: $8.7 \pm 4.0$ days in type 1 diabetes patients and $8.5 \pm 2.9$ days in type 2 diabetes patients.

The continuous intravenous insulin infusion was administered significantly longer in patients with type 1 diabetes than in type 2 diabetes: $2.7 \pm 1.6$ and $2.3 \pm$ 0.8 days, respectively $(p<0.05)$. During CIVII, patients with type 1 diabetes had a lower daily insulin requirement than patients with type 2 diabetes (45 \pm 19 and $56 \pm 22 ; p<0.01$ ), but in terms of daily insulin requirement per $\mathrm{kg}$ of body mass, the between-group difference was not statistically significant: in type 1 diabetes it was $0.63 \pm 0.21 \mathrm{U} / \mathrm{day} / \mathrm{kg}$ and in type 2 diabetes $-0.67 \pm 0.26 \mathrm{U} / \mathrm{day} / \mathrm{kg}$.

Mean blood glucose values during CIVII in both groups of patients are shown in Figure 1. In both groups, the intravenous infusion of insulin was similarly effective in reducing the glycaemia; blood glucose levels achieved in the second day of intravenous insulin infusion did not differ significantly from the values measured in subsequent days.

The primary risk factor for hypoglycaemia was type 1 diabetes (hypoglycaemia was reported in $48 \%$ of patients with type 1 diabetes and in $20 \%$ of patients with type 2 diabetes; $p<0.001$ ).

Among the patients with type 1 diabetes, those with hypoglycaemia had significantly longer diabetes duration than the patients who did not experience hypoglycaemia during the continuous infusion of insulin
(14.4 \pm 10.4 vs. $6.8 \pm 5.6$ years; $p<0.01)$. The degree of diabetes decompensation, coexistence of vascular complications, renal, gastrointestinal or cardiovascular disease, the amount of insulin administered per body weight and medicines taken before or during hospitalization did not affect the occurrence of hypoglycaemia.

In both subgroups of diabetic patients, the first episode of hypoglycaemia during CIVII occurred at similar time point. In type 1 diabetes, the first hypoglycaemia was noted at $1.4 \pm 0.9$ days of infusion and in type 2 diabetes - at $1.6 \pm 0.7$ days.

In the group of type 1 diabetic patients, those in whom hypoglycaemia occurred during CIVII were different from those who did not experience this complications in terms of the time from the diagnosis of diabetes (14.4 \pm 10.4 vs. $6.8 \pm 5.6$ years, respectively; $p<0.01)$, but were not different in terms of the duration of intravenous infusion of insulin $(2.5 \pm 0.8$ vs. $3.0 \pm$ \pm 2.1 days), total daily insulin dose $(42 \pm 16$ vs. $49 \pm$ $\pm 23 \mathrm{U} /$ day), daily insulin dose per $\mathrm{kg}$ of body weight $(0.60 \pm 0.16$ vs. $0.66 \pm 0.26 \mathrm{U} / \mathrm{day} / \mathrm{kg})$ as well as in terms of age (39.8 \pm 15.2 vs. $35.2 \pm 12.9$ years), body weight $(69.0 \pm 9.0$ vs. $17.5 \pm 17.3 \mathrm{~kg})$ and BMI $(24.2 \pm 3.6$ vs. $\left.25.8 \pm 5.1 \mathrm{~kg} / \mathrm{m}^{2}\right)$; although a trend toward lower body mass was observed in the group with hypoglycaemia.

In the multiple regression model with hypoglycaemia as a dependent variable and the age, time from diagnosis of diabetes, $\mathrm{BMI}, \mathrm{HbA}_{1 \mathrm{c}^{\prime}}$ plasma creatinine and daily insulin dose per $\mathrm{kg}$ of body weight as inde- 
pendent variables, the duration of diabetes was the significant risk factor for hypoglycaemia (regression coefficient $B=0.398 ; p<0.05$ ).

Unlike patients with type 1 diabetes, the patient with type 2 diabetes who had hypoglycaemia did not differ significantly from those who did not experience this complication in terms of the time from the diagnosis of diabetes (10.4 \pm 9.1 vs. $10.1 \pm 8.3$ years), duration of intravenous infusion of insulin ( $2.2 \pm 0.7$ vs. $2.3 \pm 0.8$ days $)$, age $(62.2 \pm 13.7$ vs. $60.5 \pm 11.8)$, body weight $(79.3 \pm 19.1$ vs. $86.7 \pm 19.6 \mathrm{~kg})$ and BMI $\left(29.6 \pm 6.6\right.$ vs. $\left.31.3 \pm 6.1 \mathrm{~kg} / \mathrm{m}^{2}\right)$. Significant differences were, however, observed in total daily dose of insulin $(45 \pm 14$ vs. $58 \pm 22 \mathrm{U} /$ day; $p<0.001)$ and daily insulin dose per $\mathrm{kg}$ of body weight $(0.56 \pm 0.11$ vs. $0.69 \pm 027 \mathrm{U} /$ day/kg; $\mathrm{p}<0.01$ ), which indicates that people who experienced hypoglycaemia required lower doses of insulin. Also in type 2 diabetes patient, there is a noticeable trend towards lower body mass (more precisely - being less overweight) in the group of patients in whom hypoglycaemia had occurred. Multiple regression model with hypoglycaemia as a dependent variable and age, the time from diagnosis of diabetes, $\mathrm{BMI}, \mathrm{HbA}_{1 c^{\prime}}$ plasma creatinine and daily insulin dose per $\mathrm{kg}$ of body weight as independent variables showed that the daily insulin dose per $\mathrm{kg}$ of body weight was a significant risk factor $(B=-0.23$; $p<0.05)$ for hypoglycaemia.

\section{Discussion}

Hypoglycaemia is a common health problem in diabetic patients, especially those treated with insulin [9]. Nowadays, hypoglycaemia is considered as a particularly unfavourable event in the course of diabetes treatment $[4,6]$. Its occurrence has been associated with the treatment of diabetes since the introduction of insulin, almost 100 years ago, and until recently it has been considered as an inevitable part of diabetes therapy.

The fundamental reason of frequent occurrence of hypoglycaemia is very narrow therapeutic index of insulin. This means that the difference between the therapeutic dose of insulin and its harmful dose - that is, causing hypoglycaemia - is very small. This in turn is due to the fact that targets for glycaemic control in diabetic patients are very close to diagnostic threshold for hypoglycaemia, which is now set at $<70 \mathrm{mg} / \mathrm{dL}$ [4]. This value was announced only in 2014; whereas at the time when the study was conducted the recommended threshold for the diagnosis of hypoglycaemia was $<60 \mathrm{mg} / \mathrm{dL}$.

There are many reasons to avoid hypoglycaemia as much as possible. It affects unfavourably the car- diovascular and central nervous systems. Even mild hypoglycaemic episodes, if they are frequent, can lead to significant intellectual disability, especially in the elderly [10-12]. Indirect evidence of the nature of the hypoglycaemic problem is the fact that all new medications introduced in the last decade (incretins, SGLT-2 inhibitors) do not pose the risk of hypoglycaemia due to their mechanism of action [6], and all new insulin preparations (e.g. insulin glargine, degludec) are characterized by a lower risk of hypoglycaemia than the insulins used so far.

The way to reduce the risk of hypoglycaemia is to identify the factors leading to its increase and avoid them. Therefore, the aim of this study is to identify these factors, focusing on patients treated with intravenous insulin infusion in hospital setting, because in this group of patients the risk of developing hypoglycaemia is the greatest and its consequences may be the most dangerous. Obviously, the population of hospitalized patients is different from the outpatients, but it cannot be ruled out that those who have hypoglycaemia during hospitalization are also at risk of this complication outside the hospital. Given the impact of physical activity on the risk of hypoglycaemia and the fact that during hospitalization, the physical activity is low, the risk factors for hypoglycaemia should be even more important outside the hospital, where physical activity may additionally decrease blood glucose. Monitoring the risk of hypoglycaemia in hospital setting where insulin can be administered intravenously also allowed for a more direct assessment of hypoglycaemia, because insulin was injected immediately into circulating blood and a significant barrier to its action (i.e. the need to absorb insulin from subcutaneous tissue into the blood) was eliminated.

The intravenous administration of insulin is the most effective method of treating hyperglycaemia, because a reduction in glycaemia is achieved in all patients [7, 13]. In addition, the intravenous infusion of insulin allows for precise regulation of the drug supply - the half-time of insulin molecule is several minutes, and in the case of hypoglycaemia, intravenous infusion allows for immediate stoppage of progressive decrease in glycaemia. Therefore - paradoxically - the most effective method of lowering glycaemia is also the safest. The intravenous insulin infusion is a commonly used method in hospital settings [14]. In patients hospitalized for acute diabetic complications characterized by hyperglycaemia (ketoacidosis, hyperosmolar state, lactic acidosis) and other severe conditions associated with rapid increase in blood glucose, this is a treatment of choice [15-17]. However, this method is also commonly used in chronic decompensation of diabetes 
- as in patients taking part in this study. The intravenous insulin infusion not only provides desired degree of metabolic control of diabetes, but also allows for determination of, for example, insulin requirement per day, at meal times and at night. It is quite often used for this purpose in diabetic patients. It can be safe, provided that the risk of hypoglycaemia is minimalized, especially considering that the aim of intravenous insulin therapy is to prevent both significant hyperglycaemia and hypoglycaemic episodes. One of the basic methods of avoiding specific events is to identify risk factors for their occurrence. Therefore, determining what is the profile of patients that are most likely to have hypoglycaemia during intravenous insulin therapy is critical to reducing the risk of developing this acute complication of glucose-lowering treatment.

In hospital conditions, the intravenous infusion of insulin, its flow rate and duration is defined by physicians, but the preparation for insulin infusion, its administration and adjustment during administration are among the tasks of the nursing staff $[18,19]$. This was one of the basic prerequisites for this study. It can be assumed that if a nurse who prepares an infusion of insulin and connects the infusion set to a patient is aware that a given patient is at increased risk of hypoglycaemia, she or he may monitor the intravenous insulin infusion more carefully and give the patient maximum attention, which would undoubtedly help to reduce the risk of hypoglycaemia. For this reason, the results of the study are of great practical importance.

The study showed that the highest risk for hypoglycaemia was observed in patients with type 1 diabetes, especially those who have had diabetes for more than 10 years. This is not an unexpected observation. In type 1 diabetes, insulin resistance, or disturbed insulin action, is not an issue; therefore, the effect of insulin is very directly proportional to the dose and very easy to measure. In patients with long-lasting type 1 diabetes, also the endogenous glucagon production is impaired, hence their ability to defend against hypoglycaemia is very limited $[20,21]$.

Interestingly, no association was found between the occurrence of hypoglycaemia and factors such as the degree of decompensation of diabetes, coexistence of vascular complications, renal disease, gastrointestinal and cardiovascular diseases, the amount of insulin administered per body weight or drugs used. This means that intravenous infusion of insulin by skilled and experienced nursing stuff (such as the nurses employed in a specialist hospital where the study was conducted) effectively eliminates the risk of hypoglycaemia in situations where the risk is particularly high, e.g. in renal failure. However, the professional qualifications and experience of the staff is not sufficient to offset the effects of long-term type 1 diabetes or type 1 diabetes in general. In this type of diabetes, hypoglycaemia was more than twice as common as in type 2 diabetes.

The study found that the first episode of hypoglycaemia occurred most often in the second day of intravenous infusion, both in patients with type 1 and type 2 diabetes. This is easily explained by the fact that the basic principle of insulin therapy, regardless of the route of administration, is to start with small doses and gradually increase them. In the second day of the infusion, the insulin dose was already higher, and the glycaemic values were lower than the first, hence the patients were more likely to have hypoglycaemia. This also means that special attention should be paid in the second day of intravenous insulin therapy, when hypoglycaemia is most prevalent.

The greatest value of the study is, however, the identification of the most significant risk factors for the occurrence of hypoglycaemic episodes during CIVII. In patients with type 1 diabetes this is a long-term duration of diabetes, and in type 2 diabetes - relative insulin sensitivity, in other words, less insulin resistance.

Similar conclusions can be found in recently published papers and recommendations describing the pathophysiology, prevention and management of hypoglycaemia [18, 22-27]. Clinical practice also indicates that patients who have had type 1 diabetes for more than 10 years are at the highest risk for hypoglycaemia. However, most of publications primarily focus on outpatient treatment, and hypoglycaemia in the context of hospitalization is most often related to patients admitted to hospital due to severe hypoglycaemia (associated with loss of consciousness) or development of hypoglycaemia in hospitalized critically-ill patients. In the latter group, hypoglycaemic episodes indicate poor prognosis and are a symptom of progressive multi-organ failure. Our study included patients in good general condition who have had poorly glycaemic control for a long time (mean $\mathrm{HbA}_{1 \mathrm{c}}$ was approximately $10 \%)$ and who were scheduled to reduce glycaemia by administering insulin intravenously for 1-3 days. This type of treatment is rarely studied in the literature for the risk of hypoglycaemia. In this context, the reliable identification of risk factors, also in the multiple regression model, offers the possibility of introducing such protocols for intravenous infusion of insulin that minimize the risk of developing hypoglycaemia.

In conclusion, the study showed that intravenous insulin therapy in patients with chronically decompensated diabetes mellitus is associated with hypoglycaemia in $50 \%$ of patients with type 1 diabetes and in every fifth patient with type 2 diabetes. Among type 1 diabetes patients, those with long-term disease 
duration are most likely to suffer from hypoglycaemia, whereas in patients with type 2 diabetes the risk of hypoglycaemia is significantly higher among those with lower degree of insulin resistance. In order to prevent the hypoglycaemic episodes during intravenous insulin infusion, physicians and nurses using intravenous insulin therapy should closely monitor the patients undergoing intravenous treatment, particularly type 1 diabetes patients with long-term diabetes duration and patients with type 2 diabetes with a low degree of overweight and/or low daily insulin requirement, i.e. clinical features of low insulin resistance.

\section{REFERENCES}

1. Gajewska M, Gebska-Kuczerowska A, Gorynski P, et al. Analyses of hospitalization of diabetes mellitus patients in Poland by gender, age and place of residence. Ann Agric Environ Med. 2013; 20(1): 61-67, indexed in Pubmed: 23540213.

2. Schneider ALC, Kalyani RR, Golden S, et al. Diabetes and Prediabetes and Risk of Hospitalization: The Atherosclerosis Risk in Communities (ARIC) Study. Diabetes Care. 2016; 39(5): 772-779, doi: 10.2337/dc15-1335, indexed in Pubmed: 26953170.

3. Li TC, Kardia SLR, Li Cl, et al. Glycemic control paradox: Poor glycemic control associated with higher one-year and eight-year risks of all-cause hospitalization but lower one-year risk of hypoglycemia in patients with type 2 diabetes. Metabolism. 2015; 64(9): 1013-1021, doi: 10.1016/j.metabol.2015.05.004, indexed in Pubmed: 26026367.

4. Polskie Towarzystwo Diabetologiczne. Zalecenia kliniczne dotyczące postępowania z chorymi na cukrzycę. Stanowisko Polskiego Towarzystwa Diabetologicznego. Clin Diabetol. 2017; 6(supl. A): A30-A31.

5. Tattersall RB. Frequency, cause and treatment of hypoglycaemia. W: Frier B.M., Fisher B.M. (red.) Hypoglycaemia in Clinical Diabetes. Wiley: Chichester; 1999: 55-87.

6. Polskie Towarzystwo ogiczne. Zalecenia kliniczne dotyczące postępowania z chorymi na cukrzycę. Stanowisko Polskiego Towarzystwa ogicznego. Clin Diabetol. 2017; 6(supl. A): A20-A23.

7. Ruxer J, Chromińska-Szosland D, Bukowczyk P, et al. Porównanie efektywności intensywnej insulinoterapii z wykorzystaniem dożylnej i podskórnej pompy insulinowej. Diabet Pol. 1995; 2: 24-27.

8. Dupuy O, Mayaudon H, Palou M, et al. Optimized transient insulin infusion in uncontrolled type 2 diabetes: evaluation of a pragmatic attitude. Diabetes Metab. 2000; 26: 371-375.

9. Frier BM, Jensen MM, Chubb BD. Hypoglycaemia in adults with insulin-treated diabetes in the UK: self-reported frequency and effects. Diabet Med. 2016; 33(8): 1125-1132, doi: 10.1111/ /dme.12878, indexed in Pubmed: 26248501.

10. Allen KV, Pickering MJ, Zammitt NN, et al. Effects of acute hypoglycemia on working memory and language processing in adults with and without type 1 diabetes. Diabetes Care. 2015;
38(6): 1108-1115, doi: 10.2337/dc14-1657, indexed in Pubmed: 25758768.

11. Feinkohl I, Price JF, Strachan MWJ, et al. The impact of diabetes on cognitive decline: potential vascular, metabolic, and psychosocial risk factors. Alzheimers Res Ther. 2015; 7(1): 46, doi: 10.1186/ /s13195-015-0130-5, indexed in Pubmed: 26060511.

12. Moheet A, Seaquist ER. Hypoglycemia as a driver of cardiovascular risk in diabetes. Curr Atheroscler Rep. 2013; 15(9): 351, doi: 10.1007/s11883-013-0351-7, indexed in Pubmed: 23881546.

13. Blackshear PJ, Roussell AM, Cohen AM, et al. Basal-Rate Intravenous Insulin Infusion Compared to Conventional Insulin Treatment in Patients With Type II Diabetes: A Prospective Crossover Trial. Diabetes Care. 1989; 12(7): 455-463, doi: 10.2337/diacare.12.7.455.

14. Krzymień J. Dożylne wlewy insuliny podczas hospitalizacji. Przew Lek. 2007; 4: 73-79.

15. Furnary AP. Insulin infusions for cardiac surgery patients with diabetes: a call to reason. Endocr Pract. 2002; 8(1): 71-72, doi: 10.4158/EP.8.1.71, indexed in Pubmed: 11939767.

16. van den Berghe $G$, Wouters $P$, Weekers $F$, et al. Intensive insulin therapy in critically ill patients. N Engl J Med. 2001; 345(19): 1359-1367, doi: 10.1056/NEJMoa011300, indexed in Pubmed: 11794168.

17. Goldberg PA, Siegel MD, Sherwin RS, et al. Implementation of a Safe and Effective Insulin Infusion Protocol in a Medical Intensive Care Unit. Diabetes Care. 2004; 27(2): 461-467, doi: 10.2337/ diacare.27.2.461.

18. Strojek K. Hipoglikemia. W: Cukrzyca (red. J. Sieradzki), wyd. 2. Via Medica, 2016, Gdańsk: 539-542.

19. Szewczyk A. Pielęgniarstwo diabetologiczne. PZWL, Warszawa 2013: 115-126.

20. Samson WK, Stein LM, Elrick M, et al. Hypoglycemia unawareness prevention: Targeting glucagon production. Physiol Behav. 2016; 162: 147-150, doi: 10.1016/j.physbeh.2016.04.012, indexed in Pubmed: 27080082.

21. Martín-Timón I, Del Cañizo-Gómez FJ. Mechanisms of hypoglycemia unawareness and implications in diabetic patients. World J Diabetes. 2015; 6(7): 912-926, doi: 10.4239/wjd.v6.i7.912, indexed in Pubmed: 26185599.

22. Wierzchowska A, Zozulińska-Ziółkiewicz D. Hipoglikemia w cukrzycy typu 1. Diabet Prakt. 2011; 12(6): 210-215.

23. Zammitt NN, Frier BM. Hypoglycemia in type 2 diabetes. Diabetes Care. 2005; 28: 2948-2961.

24. Allen KV, Frier BM. Nocturnal hypoglycemia: clinical manifestations and therapeutic strategies toward prevention. Endocr Pract. 2003; 9(6): 530-543, doi: 10.4158/EP.9.6.530, indexed in Pubmed: 14715482.

25. Sircar M, Bhatia A, Munshi M. Review of Hypoglycemia in the Older Adult: Clinical Implications and Management. Can J Diabetes. 2016; 40(1): 66-72, doi: 10.1016/j.jcjd.2015.10.004, indexed in Pubmed: 26752195.

26. International Hypoglycaemia Study Group. Minimizing Hypoglycemia in Diabetes. Diabetes Care. 2015; 38(8): 1583-1591, doi: 10.2337/dc15-0279, indexed in Pubmed: 26207052.

27. Seaquist ER, Anderson J, Childs B, et al. Hypoglycemia and diabetes: a report of a workgroup of the American Diabetes Association and the Endocrine Society. Diabetes Care. 2013; 36(5): 1384-1395, doi: 10.2337/dc12-2480, indexed in Pubmed: 23589542. 